\title{
Towards observations of Population III galaxies
}

\author{
Daniel Schaerer and Roser Pelló \\ Laboratoire d'Astrophysique, Observatoire de Midi-Pyrénées, \\ 14 Avenue E. Belin, F-\$1400 Toulouse, la France
}

\section{Introduction}

We are undertaking various studies of primordial galaxies with the following main aims:

(i) synthesize the properties of rest-frame UV-optical spectra of Population III galaxies and 'transition objects' between Pop III and normal starbursts;

(ii) devise optimal search strategies for the detection of Pop III and related objects with ground-based and space observations;

(iii) simulate photometric and spectroscopic observations of Pop III galaxies to quantify the feasibility of such studies especially for future ground-based instruments; and

(iv) study the physics of Pop III and 'transition' galaxies and their implications on cosmology and the physics of galaxies.

\section{Ongoing research on distant galaxies and Population III objects}

Recent synthesis models of Schaerer $(2002,2003)$ predicting the detailed SEDs of Pop III (metallicity $Z=0$ ) objects and starbursts from very low $\left(Z=10^{-7}\right)$ to 'normal' (currently observed) metallicities are used as a base. For illustration we show a predicted Pop III SED and the predicted Ly $\alpha$ equivalent width at metallicities $Z=0,10^{-7}, 10^{-5}$ to solar in Figure 1 .

The SED shows the characteristic features of Pop III and very metal-poor galaxies with ongoing active star formation: the presence of strong emission lines including He II recombination lines, due to the exceptional hardness of the ionising spectrum (Tumlinson \& Shull 2000; Schaerer 2002, 2003). In principle both of the strongest lines (Ly $\alpha, \mathrm{He} I \mathrm{I} 1640$ ) are detectable from objects with redshifts $z \gtrsim 6-8$ with sufficient exposure times ( $c f$. Schaerer \& Pelló 2002a and in preparation). However, given the various effects which can potentially reduce Ly $\alpha$ (dust, geometrical/velocity structure, absorption by $\mathrm{H}$ I for objects beyond re-ionisation), He II $\lambda 1640$ may turn out to be the strongest emission line in very distant objects.

The predicted maximum or average of $W(\operatorname{Ly} \alpha)$ - to be taken as upper limits due to the effects just mentioned - shows a relatively minor increase at metallicities $0.02 \lesssim Z / \mathrm{Z}_{\odot} \lesssim 1$ but much larger values at metallicities $10^{-5} \gtrsim Z \geq 0$ (Schaerer 2003). We note that young populations with very low metallicities $\left(Z \simeq 10^{-4 \ldots-7}\right)$ can rival with the high median Ly $\alpha$ equivalent width $(\sim 430 \AA)$ 

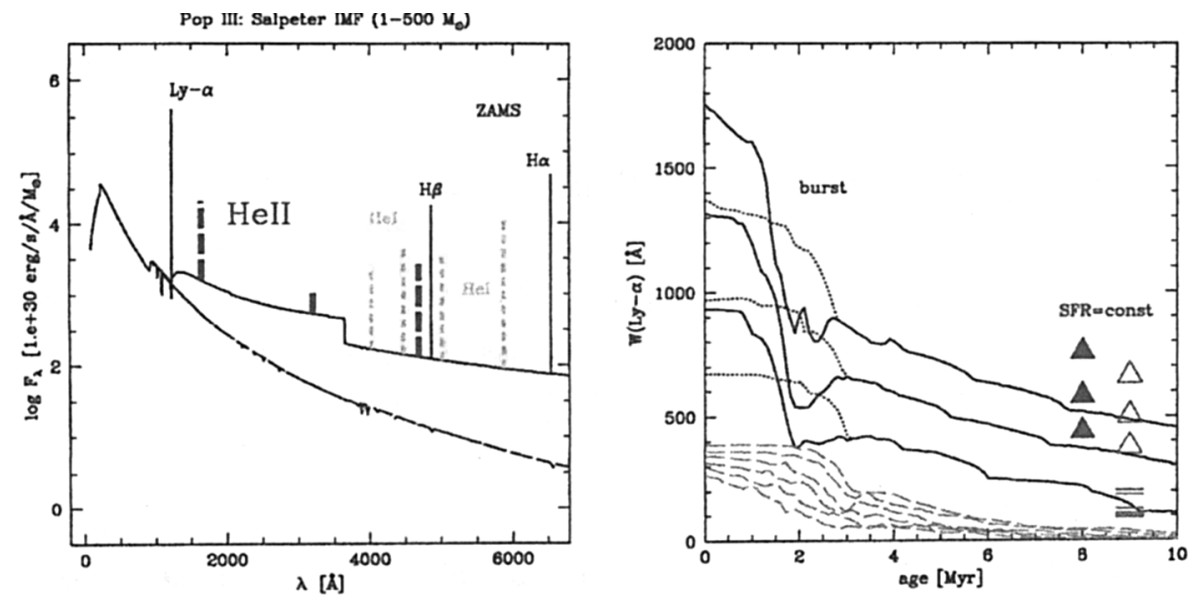

Figure 1. Predicted Pop III SED (left) and temporal evolution of $W(\mathrm{Ly} \alpha)$ at metallicities from $Z=0$ to solar (right). A Salpeter IMF with $M_{\mathrm{up}} \simeq 100 \mathrm{M}_{\odot}$ (dashed and dotted lines) or $500 \mathrm{M}_{\odot}$ (left panel and solid lines) is adopted.

of the objects found in the Large Area Lyman Alpha (LALA) survey of Malhotra $\&$ Rhoads (2002) at $z=4.5$. If such metallicities are considered, there seems no immediate need for AGN, extreme IMFs, or even Pop III objects as argued by these and other authors.

The expected photometric properties of Pop III objects have been briefly discussed in Schaerer \& Pelló (2002b). Simulations of near-IR spectroscopic observations with $10 \mathrm{~m}$ class telescopes have been presented in Schaerer \& Pelló (2002a). A more detailed account will be given in Pelló \& Schaerer (in preparation).

Other recent/ongoing investigations based on our synthesis model predictions include the study of Lyman- $\alpha$ emission and absorption in observed highredshift galaxy samples (Valls-Gabaud \& Schaerer, in preparation). We are also investigating the $\mathrm{H}$ and $\mathrm{He}$ II re-ionisation history of the Universe with a combined chemical evolution model (Ferrara \& Schaerer, in preparation).

\section{References}

Malhotra, S., Rhoads, J.E. 2002, ApJ (Letters) 565, L7

Schaerer, D. 2002, A\&A 382, 28

Schaerer, D. 2003, A\&A 397, 527

Schaerer, D., Pelló, R. 2002a, in: J. Bergeron \& G. Monnet (eds.), Scientific Drivers for ESO Future VLT/VLTI Instrumentation, ESO Astrophysics Symposia (Berlin: Springer), p. 48

Schaerer, D., Pelló, R. 2002b, in: M. Sauvage, G. Stasinska, L. Vigroux, D. Schaerer \& S. Madden (eds.), The Evolution of Galaxies. II. Basic Building Blocks, Ap\&SS 281,475

Tumlinson, J., Shull, J.M. 2000, ApJ (Letters) 528, L65 